\title{
Redefining the Therapeutic Wheel of Diabetes Management
}

\author{
Manish Maladkar*, Shrikant Patil and Heta Viswanathan \\ 23-A Shah Industrial Estate, Andheri, India
}

Submission: August 09, 2018; Published: September 17, 2018

*Corresponding author: Manish Maladkar, 23-A Shah Industrial Estate, Andheri, Mumbai, India, Email: scientific@aristopharma.org

\begin{abstract}
Diabetes is one of the biggest global health emergencies of the 21st century. As per the International Diabetes Federation (IDF), approximately 50\% of all people with diabetes live in just three countries: China (109.6 million), India (69.2 million) and the USA (29.3 million). According to ICMR-INDIAB National study, the Indian Diabetic study, around 62.4 million people had diabetes in 2011 and 77 million had prediabetes. Many Asian races show a tendency for fat deposition in the abdominal area which is known as central adiposity. Hyperinsulinemia and insulin resistance are closely associated with central adiposity. Traditionally diagnosis of diabetes was done by measuring FPG (Fasting Plasma Glucose) and OGTT (Oral Glucose Tolerance Test) is often despite several international guidelines recommending HbA1C to diagnose diabetes. However, HbA1c cannot be used as sole criteria for diagnosis of diabetes in Indian settings, but it can be used in settings where an appropriate standardization method is available.

Effectively managing diabetes is the key to reducing the microvascular as well as macrovascular complications associated with it. Diabetes can be managed by utilizing a conjunction of non-pharmacological and pharmacological options like various classes of antidiabetic agents as guided by various national and international guidelines. Despite these efforts diabetes management practices in India remain less than satisfactory. This is partly due to diverse religions, cultures, languages, food habits, lifestyles, and traditions that impact management practices for diabetes. A need for an Indian patient specific guideline and protocol for effectively managing diabetes prompted the Research Society for the Study of Diabetes in India (RSSDI) to formulate guidelines suiting the Indian patients taking in consideration the cost effectiveness of the treatment. This article highlights and redefines the therapeutic wheel of diabetes that addresses individualizing therapy for management of diabetes.

Keywords: Diabetes; Pre-diabetes; Obesity; Antidiabetic agents; Diabetic wheel

Abbreviations: IDF: International Diabetes Federation; FPG: Fasting Plasma Glucose; OGTT: Oral Glucose Tolerance Test; RSSDI: Research Society for the Study of Diabetes in India; WHO: World Health Organization; CAD: Coronary Artery Disease; BMI: Body Mass Index; WC: Waist Circumference; WHR: Waist to Hip Ratio; FPG: Fasting Plasma Glucose; 2-h PPG: 2-h Post-Prandial plasma Glucose; Sus: Sulfonylureas; GLP: Glucagon-Like Peptide; PKA: Protein Kinase-A; AGI: Alpha Glucosidase Inhibitor; FMD: Flow-Mediated Dilatation; SMBG: Self-Monitoring Blood Glucose
\end{abstract}

\section{Introduction}

One of the biggest global health emergencies of the 21st century is Diabetes. Every year many, people live with this condition, which can result in life-changing complications. Currently, across the globe there are 415 million adults who are estimated to have diabetes, and around 318 million adults have impaired glucose tolerance, which is a risk factor for development of diabetes in the future. Around more than $80 \%$ of this 184 million people with diabetes in this age group live in low- and middle-income countries. India, the second largest country in terms of world population, has been severely affected by the global diabetes epidemic. As per the International Diabetes Federation (IDF), approximately $50 \%$ of all people with diabetes live in just three countries: China (109.6 million), India (69.2 million) and the USA (29.3 million) [1]. According to ICMR-INDIAB National study, the Indian Diabetic study around 62.4 million people had diabetes in 2011 and 77 million had pre-diabetes [2]. According to the World Health Organization (WHO) criteria, the prevalence of known diabetes was $5.6 \%$ and $2.7 \%$ among urban and rural areas, respectively [3]. The prevalence of Type 2 diabetes is increasing in migrant Indians as reported from several studies. Currently, in urban metros of India the prevalence of diabetes is fast approaching the figures commonly reported in the affluent migrant Indians.

There are strong evidences stating that Indians are prone to a greater degree of insulin resistance and a stronger genetic predisposition to diabetes. Obesity, especially central obesity and increased visceral fat due to physical inactivity, and consumption of a high-calorie/high-fat and high sugar diets are major contributing factors for the development of T2DM [4]. Most patients with type 2 diabetes are obese, and the global epidemic 
of obesity largely explains the dramatic increase in the incidence and prevalence of type 2 diabetes over the past 20 years. Obesity in Type II diabetic patients is a very common phenomenon and often termed as "Diabesity". The association of obesity with type 2 diabetes is complex and compounded by many heterogeneous factors. Obesity not only is a risk factor for development of diabetes, but also complicates the management of the disease.

Increased predisposition to diabetes \& premature CAD (Coronary Artery Disease) has been attributed to the Asian Indians generally having lower BMI than many other races but the association of Body Mass Index (BMI) with glucose intolerance is as strong as in any other population. "Asian Indian Phenotype" characterized by less of generalized obesity measured by BMI \& greater central body obesity as shown by greater Waist Circumference (WC) \& Waist to Hip Ratio (WHR) [5]. Recent studies have established lower limits for ideal BMI for Asian populations. It was shown for urban Indian population that at a BMI of $>23 \mathrm{~kg} / \mathrm{m} 2$ the risk for diabetes was significantly higher for both genders. The healthy BMI for an Indian is definitely below $23 \mathrm{~kg} / \mathrm{m} 2$. This has been confirmed by studies from other parts of India and in-migrant Indians and also from other Asian populations. According to the World Health Organization (WHO) recommendations a BMI of $18.5-22 \mathrm{~kg} / \mathrm{m} 2$ has been considered as healthy for Asian populations. Many Asian races show a tendency for fat deposition in the abdominal area which is known as central adiposity. Hyperinsulinemia and insulin resistance are closely associated with central adiposity [6]. However, it may be conceptualized that adoption of sound methods to increase awareness through diabetes education, behavioral changes and promotion of healthy lifestyle together with continuous efforts to screen for diabetes and healthy town planning may blunt the diabetes growth curve and curb the second epidemic of diabetes that is likely to occur in 2-3 decades [7].

\section{Screening and Diagnosis of Type 2 diabetes}

Type 2 diabetes is characterized by an asymptomatic phase between the actual onset of diabetic hyperglycemia and clinical diagnosis. This phase has been estimated to last at least 4-7 years, and 30-50\% cases of type 2 diabetic patients remained undiagnosed. This leads to the development of chronic complications of diabetes, which still remain a major hurdle in diabetic care, and which cause a lack of fitness to work, disability, and premature death. Therefore, early diagnosis is important for the prevention of diabetic complications.

Diabetes may be diagnosed based on HbA1C criteria or plasma glucose criteria, either the Fasting Plasma Glucose (FPG) or the 2-h Post-Prandial plasma Glucose (2-h PPG) value after a 75-g Oral Glucose Tolerance Test (OGTT). The same tests are used to both screen for and diagnose diabetes. Diabetes could be identified anywhere along the spectrum of clinical scenarios: in seemingly low-risk individuals who happen to undergo glucose testing, in symptomatic patients, and in higher-risk individuals. These tests also help in detecting individuals with prediabetes [8].
Traditionally measuring FPG and OGTT is often considered for diagnosis, despite several international; guidelines recommending HbA1C to diagnose diabetes. According to RSSDI 2015 recommendations for diagnosis of diabetes $\mathrm{HbA1}$ c cut off point of $6.5 \%$ is optimal for diagnosis of diabetes in Indian patients. However, HbA1c cannot be used as sole criteria for diagnosis of diabetes in Indian settings, but it can be used in settings where an appropriate standardization method is available [9].

\section{Management of Diabetes}

The goals in caring for patients with diabetes mellitus are to eliminate symptoms and to prevent, or at least slowdown, the development of microvascular and macrovascular complications. Microvascular (i.e., eye and kidney disease) risk reduction is accomplished through control of glycaemia and blood pressure; macrovascular (i.e., coronary, cerebrovascular, peripheral vascular) risk reduction, through control of lipids and hypertension, smoking cessation, and aspirin therapy; and metabolic and neurologic risk reduction, through control of glycaemia [10]. Glycemic management in type 2 diabetes mellitus has become increasingly complex and to some extent controversial. With a widening array of pharmacological agents now available, mounting concerns about their potential adverse effects and new uncertainties regarding the benefits of intensive glycemic control on macrovascular complications have been on rise [11].

\section{Non-Pharmacological Approaches}

Exercise and weight reduction have known to decrease insulin resistance and increase glucose tolerance. Studies have shown that there was significant reduction in the incidence of type $2 \mathrm{DM}$ with a combination of maintenance of BMI of $25 \mathrm{~kg} / \mathrm{m} 2$, eating high fiber unsaturated fat and diet low in saturated and trans-fats and lower glycemic index, regular exercise, abstinence from smoking and moderate consumption of alcohol suggesting that majority of type $2 \mathrm{DM}$ can be prevented by lifestyle modification. Patients with type 2 DM should receive a medical nutrition evaluation; lifestyle recommendations should be tailored according to physical and functional ability [12].

Nutritional therapy remains an integral part of diabetes management. As there is no "one-size-fits-all" meal plan, ADA emphasizes on development of individualized eating plan based on individual's health needs. The primary goal of nutrition therapy is to improve health by providing calories for growth and development while achieving and maintaining optimal glycemic index and normalizing dyslipidemia. Patients with type 2 diabetes are advised that lifestyle modification by changing pattern of eating and adequate physical activity can be effective in controlling the risk factors associated with this condition. A total of $60 \mathrm{~min}$ of physical activity is recommended every day for healthy Asian Indians in view of the high predisposition to develop T2DM and Coronary Heart Disease. This should include at least $30 \mathrm{~min}$ of moderate-intensity aerobic activity, $15 \mathrm{~min}$ of work-related activity, and $15 \mathrm{~min}$ of muscle-strengthening exercises [13]. Meta- 
analyses demonstrate that lifestyle interventions, including diet and physical activity, led to a $63 \%$ reduction in diabetes incidence in those at high risk.

In spite of the underscored importance of lifestyle measures in diabetes therapy, most diabetics cannot escape the value of pharmacotherapy to achieve target glucose concentrations. Different oral hypoglycemic agents have been in use to aid in maintenance of blood glucose level at the requisite threshold in diabetics through distinct mechanisms [14].

\section{Pharmacological approaches for the management of Diabetes}

Pharmacological approaches for management of diabetes include early initiation of anti-diabetic agents associated with improved glycemic control and reduced long-term complications associated with type 2 diabetes. There are various classes of antidiabetic agents used for managing T2DM.

Biguanides: Biguanides, of which Metformin is the most commonly used in overweight and obese patients, suppresses hepatic glucose production, increases insulin sensitivity, enhances glucose uptake by phosphorylating GLUT-enhancer factor, increases oxidation of fatty acid, and decreases the absorption of glucose from the gastrointestinal tract. Metformin remains the first line anti-diabetic drug due to its properties such as being efficacious, weight neutral, economical, devoid of major sideeffects such as hypoglycemia. Due to the concern of development of lactic acidosis, Metformin should be used with caution in elderly diabetic individuals with renal impairment. It has a low incidence of hypoglycemia as compared to sulfonylureas.

Sulphonylureas: Sulfonylureas (SUs) are the oldest and most widely used medications for the treatment of T2DM. Although SU therapy effectively lowers blood glucose concentrations (average decrease in FPG of 2-4 mmol/l, accompanied by a decrease in HbA1c of $1-2 \%$ ) by stimulating insulin secretion from $\beta$-cells, treatment with SUs is associated with a progressive linear decline in $\beta$-cell function. Eventual inability to maintain glycemic control reflects an advanced stage of $\beta$-cell failure. Hypoglycemia is the most common and most serious adverse event associated with SU therapy, mainly because of insulin release being initiated even when glucose concentrations are below the normal threshold for normal physiologic glucose-stimulated insulin release. Weight gain, regarded as a class effect of SUs, is thought to result from an anabolic effect of increased insulin concentration. Owing to decreased effectiveness of SUs over time and an associated decline in the insulin secretory reserve, combination therapy has focused mainly on adding insulin-sensitizing medications, including Metformin and thiazolidinediones [15].

Glinides: This group of insulin secretagogues works through binding sites on beta cells that are both distinct and similar to those of SU. These drugs work by stimulating first-phase insulin release but not second-phase release. Thus, these drugs are less likely to produce late or fasting hypoglycemia compared with SUs. Glinides close the potassium channels of the beta cell and open the calcium channel, thus inducing insulin exocytosis. Unlike the commonly used sulfonylureas, the meglitinides have a very short onset of action and a short half-life. Repaglinide has shown similar effects on HbA1c and FPG levels when compared with Glyburide, 0.5 to $2 \%$ and 65 to $75 \mathrm{mg}$ per dL (3.6 to $4.2 \mathrm{mmol}$ per L), respectively. Some potential advantages of this class of agents include a greater decrease in postprandial glucose and a decreased risk of hypoglycemia [16].

Thiazolidinediones (Glitazones): The potent insulinsensitizing effect of the TZDs is mediated through activation of the Peroxisome Proliferator-Activated Receptor Gamma (PPAR- $\gamma$ ), a nuclear receptor that regulates the production of proteins involved in glucose and lipid homeostasis. The TZDs appear to improve insulin sensitivity via several mechanisms mediated by PPAR- $\gamma$, primarily in adipose tissue. While the pre-dominant insulin-sensitizing effects occur in skeletal muscle, these appear to be mediated indirectly via altered Free Fatty Acid (FFA) supply. The risk of hypoglycemia is negligible and may be more durable in their effectiveness against Sulphonylurea and Metformin. TZDs may reduce the burden of CVD, as indicated by their effects on both traditional and non-traditional cardiovascular risk factors, and reductions in myocardial infarction and stroke in the Proactive study [17].

Alpha-Glucosidase inhibitors: Alpha Glucosidase Inhibitor (AGI) prevents the digestion of carbohydrates including starch and table sugar, attenuates postprandial hyperglycemia and delays the development of type 2 diabetes in patients with IGT. Miglitol, an alpha GI, improves endothelial dysfunction assessed by the response of forearm blood flow to reactive hyperemia and FlowMediated Dilatation (FMD) in patients with type 2 diabetes and CAD. Acarbose, an alpha GI, improves postprandial endothelial dysfunction in patients with type 2 diabetes and reduces the risk of cardiovascular events in patients with type 2 diabetes and IGT [18]. Voglibose may also facilitate mobilisotary $\alpha$ endogenous Glycogen-Like Peptide 1 (GLP-1), which has an inhibitory action on glycogen, thus lowering fasting glucose levels too. Voglibose treatment has resulted in an increased release of GLP-1, which is an insulinotropic hormone and it has also increased release of GLP-1, which is known to enhance insulin secretion and insulin sensitivity [19].

The current oral blood glucose lowering agents and dietary measures only partially correct the multiple metabolic defects in diabetes with insulin resistance remaining relatively impervious to treatment. Hence, there is a need for newer anti-diabetic agents in the management of type 2 diabetes mellitus.

\section{Newer Agents in the management of Type 2 Diabetics}

DPP-IV inhibitors: Dipeptidyl peptidase IV (DPP-IV) inhibitors act primarily by blocking incretin degradation [inhibit the breakdown of glucagon-like peptide (GLP-1) and glucosedependent insulinotropic peptide (GIP) leading to an increase in plasma concentrations of the same]. This results in stimulation of insulin secretion, reduction in plasma glucose and glucagon 
levels, and inhibition of gastric emptying. Incretins also govern $\beta$-cell differentiation; mitogenesis and survival which is how DPPIV inhibition can preserve $\beta$-cell mass and improve their secretory function. Treatment with the DPP IV inhibitors is shown to reduce the risk of myocardial infarction (an effect dependent on the blood glucose levels) probably mediated via the GLP-1 receptor pathway and the Protein Kinase-A (PKA) signaling pathway [20].

GLP-1 Receptor Analogues: Glucagon-like peptide (GLP$1 \mathrm{RA}$ ) receptor analogues are synthesized by small intestinal $\mathrm{L}$ cells. They heighten glucose-dependent insulin secretion, reduce glucagon secretion, promote weight loss, slow gastric emptying, decrease appetite, and promote $\beta$-cell regeneration. They do not cause hypoglycemia, in the absence of therapies that otherwise cause hypoglycemia [20].

SGLT-2 Inhibitors (Sodium-glucose linked cotransporter-2): SGLT-2 inhibitors provide insulin independent glucose lowering by blocking glucose re-absorption in the proximal renal tubule by inhibiting SGLT-2. SGLT-2 inhibitors are known to provide modest weight loss and blood pressure reduction. Although there are 2 FDA approved agents for use in patients with type 2 diabetes, there are insufficient data to recommend their clinical use in type 1 diabetes at this time. SGLT2 inhibitors have the potential to reduce CV risk in patients with type 2 DM not only through beneficial effects on glycemic control but also via beneficial effects on body weight, lipids and serum uric acid. SGLT-2 inhibitors significantly reduce BP in patients with type 2 diabetes.

\section{Approach for Diabetes Management with Diabetic Wheel}

There are various national and international guidelines for clinicians for effective management of Type 2 diabetes resulting in improved patient outcomes. Despite various available guidelines, diabetes management practices in India remain less than satisfactory. Reasons have been attributed to diverse cultures, religions languages, food habits, lifestyles and traditions that impact management practices for diabetes. The phenomenon of deteriorating glycemic status attributed to the natural course of the disease and lack of stringent measures to eradicate root cause of the disease presents physicians with unique challenges in managing their patients with diabetes. Moreover, diabetes care is further hampered by increasing prevalence of diabetes related micro and macro-vascular complications and associated disorders (hypertension, obesity, metabolic syndrome and dyslipidemia), complicating the management aspects. A need for an Indian patient specific guideline and protocol for effectively managing diabetes prompted the Research Society for the Study of Diabetes in India (RSSDI) to formulate guidelines suiting the Indian patients taking in consideration the cost effectiveness of the treatment. The guidelines not only address the management of type 2 diabetes but also address the prevention of acute and chronic complications of diabetes. The Diabetic Wheel is adapted from the therapeutic wheel addresses the individualizing therapy for management of Diabetes.

\section{Individualizing therapy}

Choice of any anti-diabetic agents should take into account the patient's general health status and associated medical disorders. This patient centric approach may be referred to as the ABCD (EFGH) approach for diabetes management. As shown in the Figure 1, for any T2DM patient first line of therapy should be Metformin unless not tolerated or contraindicated.

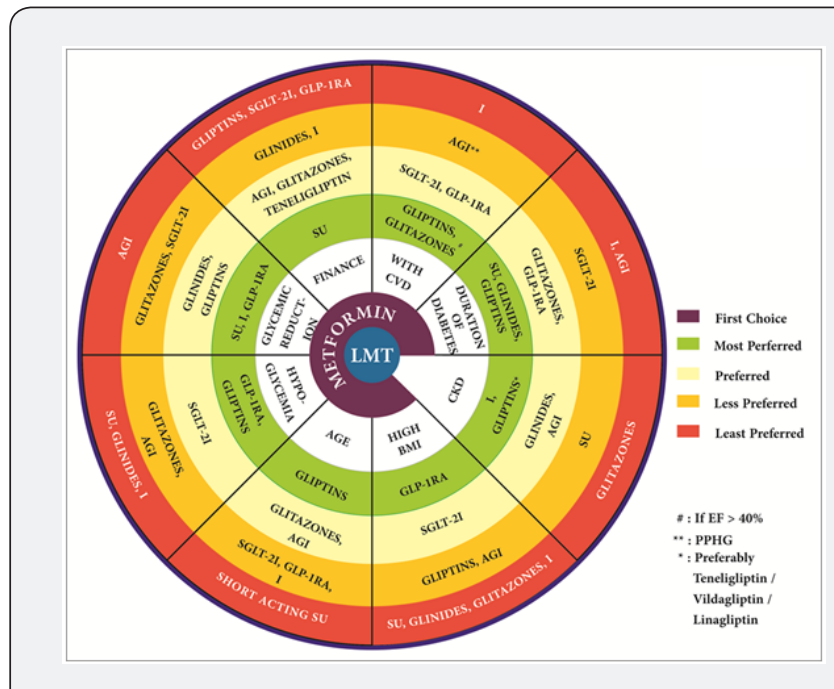

Figure 1: Therapeutic Wheel of Diabetes Management.

For a patient who has been diagnosed with diabetes consider a combination of Metformin and one of these treatment options based on Patients Age, BMI, Complications, Duration of Diabetes, Established CVD, Financial condition, Glycemic status \& Chances of Hypoglycemia.

A comparative effectiveness meta-analysis suggests that overall each new class of non-insulin agents added to initial therapy lowers HbA1c around 0.9-1.1\% [21].

\section{ABCD (EFGH) Approach}

Drug choice should be based on patient preferences as well as patients' condition, disease, and drug characteristics, with the goal of reducing blood glucose levels while minimizing side effects, especially hypoglycemia and weight gain. The approach is as follows:

\section{Age of the patient:}

- In elderly patients in order to avoid weight gain and hypoglycemia, eGFR adjusted doses of gliptins may be a suitable addition to Metformin [22].

- Agents belonging to AGI could also be important choice in elderly patients. These agents have moderate efficacy but minimal side effects.

- In elderly males, Glitazones may be a safer alternative in patients with preserved cardiac function. However, postmenopausal females must be spared for its use because of high predisposition to osteoporosis.

- While SU's, GLP-1RA, SGLT2i or Glinide should emerge as 
last choice since there are adverse effects as well as premium price associated with these agents. Benefit to risk ratio must be assessed before using these agents.

\section{High BMI}

In case of patients with high BMI, GLP-1 RA seems to be the best add on therapy. GLP-1 RA has highest weight reducing property in addition to the excellent efficacy.

- $\quad$ SGLT 2 inhibitors also have a weight reduction property albeit less than that of GLP-1 RA. SGLT-2 inhibitors have an additional advantage of excellent tolerance and can be given orally as compared to GLP-1 RA. However, their glycemic efficacy seems to be less than that of GLP-1 RA. The experience with this group of agents is less than that with GLP-1 RA [23].

- $\quad$ AGI's and Gliptins (DPP-4 Inhibitors) are weight neutral and so can be used as an add-on therapy.

- The last option for such kind of patients should be SU's, Insulin or Glitazones since they are having weight gain properties.

\section{CKD}

- A large section of diabetic population is associated with renal impairment. Appropriate choice of anti-diabetic agent is very much important in patients with renal impairment. In such patients, preference of therapy would be Gliptins as add on therapy with Metformin [24]. Few of the Gliptins need dose adjustment as per eGFR while Vildagliptin needs dose adjustment in hepatic insufficiency.

- $\quad$ Repaglinide is another agent which may be used across all stages of renal insufficiency. Similarly, Glitzones may be used in CKD; however, one has to be careful about fluid retention.

- $\quad$ Short acting sulfonylureas and AGI's may also be used across renal insufficiency; however, hypoglycemia is a huge limiting factor.

- Insulin may be used in any stages of renal insufficiency and is the best agent for this purpose.

\section{Duration of diabetes}

- As results of recent trials have suggested to utilize an aggressive approach in cases where duration of diabetes is less than 5 years, Gliptins, SU, glinide or GLP-1 RA, as an add on therapy to metformin, can be the best choices, being very potent agents. Addition of glitazones may be useful at this stage [25].

- $\quad$ SGLT-2 inhibitors may also be useful as second add on agent due to their insulin independent action which is pathophysiologically different.

- $\quad$ AGI's are last choices due to their moderate efficacy.

\section{Established CVD}

- In patients with established CVD, DPP-4 inhibitors may be preferred agents after Pioglitazone, SGLT-2 Inhibitors and AGIs because of low risk of hypoglycemia. GLP-1 analogues may be a suitable alternative for patients who are overweight or obese. AGIs may be preferred in patients with high PPHG.

- $\quad$ Pioglitazone has also been shown in different studies to reduce CVD risk.

- $\quad$ Recent data from EMPA-REG study has shown that SGLT2 inhibitors reduce CV risk and mortality, therefore may be preferred.

\section{Cost of Therapy (Financial Concern)}

- $\quad$ Cost of therapy also play an essential role considering the treatment remain continued for lifelong.

- $\quad$ SU should be first choice with Metformin by considering its cost, then after AGI or Glitazone should be used at next therapy level.

- High cost is restricting the use of Gliptins, SGLT2 inhibitors and GLP-1 RA in most of the diabetic patients.

- $\quad$ Among DPP4- Inhibitors, Teneligliptin is an exception to the prohibitive cost since this molecule in the group of Gliptins is now available at an affordable cost in India. Therefore, it can be used as a 1st add-on therapy on to Metformin.

\section{Glycemic reduction}

- Good Glycemic control of patients is directly correlated with efficacy of any anti-diabetic agent.

- Insulin followed by GLP-1RA, SUs and Glitazones have highest efficacy in terms of reducing HbA1c [26].

- $\quad$ Gliptins, SGLT2i or AGIs should be considered as add on therapy if these agents are not able to achieve glycemic target.

- It is always to be understood that good efficacy, in most cases, come with certain disadvantages as increased incidence of hypoglycemia or prohibitive cost.

\section{Hypoglycemia concerns}

- Hypoglycemia is the biggest hurdle that any medical fraternity is facing during treatment course of diabetes.

- In patients with history of hypoglycemia or for those at high risk of hypoglycemia, GLP-1 RA or gliptins should be considered as first choice with Metformin [27]. Other options include SGLT2 inhibitors, Glitazones, and AGIs.

- $\quad$ Last option for such patients should be Glinides, SU's or Insulin since there are high chances of hypoglycemia with these agents.

Group of patients where one will require avoiding hypoglycemia include:

- $\quad$ Patients with established CV disease.

- $\quad$ Elderly patients.

- $\quad$ Patients suffering from retinopathy and cannot perform 
Self-Monitoring Blood Glucose (SMBG) without help of others.

- $\quad$ Patients who stay alone, especially in remote areas.

- $\quad$ Patients who are having poor longevity.

- Patients who are having documented hypoglycemia unawareness.

- Patients with severe symptomatic hypoglycemia requiring hospitalization.

\section{Conclusion}

Given that T2D patients are heterogeneous in their clinical features and that T2D is a progressive disease, there is a clinical need for an individualized therapy that covers these issues. Personalized therapy for Indian patients with T2DM refers to the incorporation of specific patient risk factors, disease characteristics, and preferences in designing diagnostic and treatment strategies that will optimize effectiveness, safety, and adherence to therapy for a given individual. This Diabetic wheel helps the doctor in effective and strong control of diabetes by taking into consideration various factors like the age, BMI, kidney profile, duration of diabetes, co-morbid cardiovascular conditions, and risk of hypoglycemia and extent of glycemic reduction with the available anti-diabetic medications.

\section{References}

1. (2015) International Diabetes Federation. IDF diabetes atlas. In: IDF eds. A Book. $\left(7^{\text {th }}\right.$ ed), Brussels International Diabetes Federation, Belgium, Europe.

2. Anjana RM, Pradeepa R, Deepa M, Datta M, Sudha V, et al. (2011) Prevalence of diabetes and prediabetes (impaired fasting glucose and/ or impaired glucose tolerance) in urban and rural India: Phase I results of the Indian Council of Medical Research-India Diabetes (ICMRINDIAB) study. Diabetologia 54(12): 3022-3027.

3. Ramachandran A, Das AK, Joshi SR, Yajnik CS, Shah S, et al. (2010) Current Status of Diabetes in India and Need for Novel Therapeutic Agents. supplement to JAPI 58: 7-9.

4. Mohan V (2004) Why Are Indians More Prone to Diabetes. J Assoc Physicians India 52: 468-474.

5. Hetal Pandya, Lakhani JD, Patel N (2011) Obesity is becoming synonym for diabetes in rural areas of India also - an alarming situation. Int J Biol Med Res 2(2): 556-560.

6. Ramachandran A (2004) Diabetes \& obesity - The Indian angle. Indian J Med Res 120(5): 437-439.

7. Hemraj B, Chandalia (2011) Will the epidemic of diabetes in India subside. Int J Diabetes Dev Ctries 31(2): 45-47.

8. American Diabetes Association (2015) (2) Classification and diagnosis of diabetes. Diabetes Care 38 Suppl: S8-S16.
9. Int J Diabetes Ctries (October 2015)35(Suppl 1): S1-S71.

10. Khardori R, Griffing TG (2011) Type 2 diabetes mellitus treatment \& management. Medscape 16.

11. Inzucchi SE, Bergenstal RM, Buse JB, Diamant M, Ferrannini E, et al (2012) Management of hyperglycemia in type 2 diabetes: a patientcentered approach: position statement of the American Diabetes Association (ADA) and the European Association for the Study of Diabetes (EASD). Diabetes Care 35(6): 1364-379.

12. Olokoba AB, Obateru OA, Olokoba LB (2012) Type 2 Diabetes Mellitus: A Review of Current Trends. Oman Med J 27(4): 269-273.

13. Misra A, Nigam P, Hills AP, Chadha DS, Sharma V, et al. (2012) Consensus physical activity guidelines for Asian Indians. Diabetes Technol Ther 14(1): 83-98.

14. Piero MN, Nzaro GM, Njagi JM (2014) Diabetes mellitus - a devastating metabolic disorder. Asian Journal of Biomedical and Pharmaceutical Sciences 4(40): 1-7.

15. Robert GM (2010) Combination therapy for patients with Type 2 diabetes: repaglinide in combination with metformin. Expert Rev Endocrinol Metab 5(3): 331-342.

16. Luna B, Feinglos MN (2001) Oral Agents in the Management of Type 2 Diabetes Mellitus. Am Fam Physician 63(9): 1747-1756.

17. Wilding J (2006) Thiazolidinediones, insulin resistance and obesity: finding a balance. Int J Clin Pract 60(10): 1272-1280.

18. Nakamura K, Oe H, Kihara H, Shimada K, Fukuda S, et al. (2014) DPP-4 inhibitor and alpha-glucosidase inhibitor equally improve endothelial function in patients with type 2 diabetes: EDGE study. Cardiovasc Diabetol 13: 110 .

19. Dabhi AS, Bhatt NR, Shah MJ (2013) Voglibose: An Alpha Glucosidase Inhibitor. J Clin Diagn Res 7(12): 3023-3027.

20. Gude D (2012) Red carpeting the newer anti-diabetics. J Pharmacol Pharmacother 3(2): 127-131.

21. Bennett WL, Maruthur NM, Singh S, Segal JB, Wilson LM, et al. (2011) Comparative effectiveness and safety of medications for type 2 diabetes: an update including new drugs and 2-drug combinations. Ann Intern Med 154(9): 602-613.

22. (2009) Type 2 diabetes - newer agents NICE Clinical Guideline.

23. Cauthon K, Yendapally R (2015) A Review of GLP-1 Receptor Agonists and SGLT2 inhibitors for Type 2 Diabetes.

24. BMJ Blogs: Gliptins - where are we now?

25. Hanefeld M (2007) Pioglitazone and sulfonylureas: effectively treating type 2 diabetes. Int J Clin Pract Suppl (153): 20-27.

26. Gallwitz B (2014) The Future of Combination Therapies of Insulin with a Glucagon-like Peptide-1 Receptor Agonists in Type 2 Diabetes - Is it Advantageous? European Endocrinology 10(2): 98-99.

27. McCulloch DK, Nathan DM, Mulder JE (2015) Management of persistent hyperglycemia in type 2 diabetes mellitus. 
(C) This work is licensed under Creative CC Commons Attribution 4.0 License BY DOI: 10.19080/JOCCT.2018.12.555832
Your next submission with Juniper Publishers will reach you the below assets

- Quality Editorial service

- Swift Peer Review

- Reprints availability

- E-prints Service

- Manuscript Podcast for convenient understanding

- Global attainment for your research

- Manuscript accessibility in different formats

( Pdf, E-pub, Full Text, Audio)

- Unceasing customer service

Track the below URL for one-step submission https://juniperpublishers.com/online-submission.php 